\title{
Situación actual del dolor crónico en España: iniciativa "Pain Proposal"
}

\author{
A. Torralba ${ }^{1}$, A. Miquel $^{2}$ y J. Darba ${ }^{3}$ \\ ${ }^{1}$ Coordinador Nacional de Artritis (ConArtritis). ${ }^{2}$ Atención Primaria. Consejería de Sanidad de la \\ Comunidad de Madrid. ${ }^{3}$ Departamento de Economía. Universidad de Barcelona. Barcelona
}

Torralba A, Miquel A, Darba J. Situación actual del dolor crónico en España: iniciativa "Pain Proposal". Rev Soc Esp Dolor 2014; 21(1): 16-22.

\footnotetext{
ABSTRACT

One in five Europeans (19 \%) is estimated to suffer from chronic pain.

The Pain Proposal initiative represents perspectives from a range of stakeholders from across 15 European countries concerning chronic pain, including people with chronic pain, clinicians from different medical specialties, policy experts, industry members and health economists. This article sets forth the recommendations and opinions of the Pain Proposal in reference to the present chronic pain care model in Spain.

The data presented in this report have highlighted a number of inadequacies in the way chronic pain is currently managed in Spain. Patients have reported long waiting times for referral and persisting pain despite treatment, with consequences for their ability to work and their relationships with partners and family.

Healthcare professionals have also reported dissatisfaction with aspects of chronic pain management and a desire for greater support.

Improving pain management will bring substantial economic benefits: More efficient use of existing resources for pain management, less absenteeism, greater productivity, and a reduced burden on social security budgets.

Everyone involved in the chronic pain area, from healthcare professionals to government departments, needs to work together to improve pain management across Spain.
}

Key words: Chronic pain. Survey. Impact of pain. Cost of illness.

\section{RESUMEN}

Se estima que uno de cada seis españoles (17 \%) sufre de dolor crónico. La iniciativa Pain Proposal agrupó la visión de diferentes sectores involucrados en el dolor crónico procedentes de 15 países europeos, que incluyó personas con dolor crónico, diferentes especialistas médicos gestores, decisores sanitarios y economistas de la salud. En este artículo se muestran las directrices y opiniones del Pain Proposal referidas al modelo actual de atención del dolor crónico en España.

Los datos presentados en este informe han puesto de relieve una serie de deficiencias en el manejo del dolor crónico actualmente en España. Los pacientes se quejaron del largo periodo de tiempo de espera para el diagnóstico y del dolor persistente a pesar del tratamiento, lo cual afectaba a su capacidad laboral y a sus relaciones socio-familiares. Profesionales de la salud manifestaron también insatisfacción con diferentes aspectos del manejo del dolor crónico. La mejora de la gestión de dolor proporcionaría beneficios económicos sustanciales: un uso más eficiente de los recursos existentes para el tratamiento del dolor, menor absentismo, una mayor productividad y una menor carga sobre los presupuestos de la Seguridad Social.

Todos los involucrados en el área de dolor crónico, desde profesionales de la salud a los departamentos de Gobierno, deben trabajar juntos para mejorar el manejo del dolor en España.

Palabras clave: Dolor crónico. Encuesta. Impacto del dolor. Coste de la enfermedad. 


\section{INTRODUCCIÓN}

Se estima que uno de cada cinco europeos (19\%) (1) sufre dolor crónico. Aunque a nivel nacional no son muchos los estudios epidemiológicos realizados $(1,2)$, datos recientes estiman que la prevalencia es ligeramente inferior a la media europea (17\%) (3).

Actualmente el dolor crónico no se adscribe a una especialidad médica concreta, abordándose en la mayoría de los casos en atención primaria, pero siendo motivo de consulta en diferentes especialidades y disciplinas. Esto hace que a veces el proceso del enfermo, hasta que recibe el diagnóstico y el tratamiento adecuado, sea tortuoso y costoso. El dolor crónico, además de afectar considerablemente al ámbito laboral, social y familiar del enfermo, supone una carga económica considerable para el sistema sanitario $(3,4)$.

El proyecto Pain Proposal es una iniciativa desarrollada bajo el aval de la Federación Europea de la Asociación Internacional del Estudio del Dolor (EFIC) y la Federación Europea de Asociaciones Neurológicas (EFNA). Esta iniciativa surgió de la necesidad percibida entre los diferentes sectores involucrados en el dolor crónico, por identificar de forma global las carencias en su manejo e intentar coordinar los esfuerzos de todas las partes involucradas.

El principal objetivo de la iniciativa Pain Proposal fue, por tanto, lograr una visión global consensuada acerca de la repercusión del dolor crónico en toda Europa, a fin de identificar las medidas necesarias para subsanar las deficiencias actuales en la atención sanitaria de este trastorno.

La iniciativa Pain Proposal recoge el posicionamiento a título personal de numerosos expertos pertenecientes a diferentes sociedades involucradas en el diagnóstico, tratamiento y gestión del dolor crónico, procedentes de más de 15 países. En el proyecto participaron médicos especialistas, 1.472 médicos de atención primaria (100 españoles), miembros de la industria farmacéutica, economistas sanitarios y políticos, así como 2.019 pacientes con dolor crónico (201 españoles).

El presente artículo, elaborado con la colaboración de expertos españoles participantes en la iniciativa Pain Proposal, expone los resultados circunscritos al modelo actual de atención integral del dolor crónico en España. Desde la perspectiva global marcada por la iniciativa Pain Proposal, en este artículo se muestra el impacto que el dolor crónico tiene en la vida del paciente y su manejo en el sistema sanitario español que abarca aspectos tan relevantes como el proceso del diagnóstico, tratamientos y acceso a la formación especializada, así como la carga económica que el dolor crónico supone.

\section{IMPACTO DEL DOLOR CRÓNICO EN EL PACIENTE}

El dolor crónico tiene un efecto devastador en muchos aspectos de la vida diaria. No sólo disminuye la calidad de vida del paciente al repercutir negativamente en su salud física y emocional $(3,5)$. También tiene efectos adversos en ámbitos no relacionados con la salud (6) ya que dificulta participar plenamente de la vida social y familiar, y disminuye la capacidad para trabajar de manera productiva $(3,5)$.

Según una encuesta europea, la mitad de los pacientes con dolor crónico se sienten cansados todo el tiempo y el $40 \%$ indefensos o incapaces de pensar o funcionar con normalidad (1).

El resultado de la iniciativa Pain Proposal (5) al valorar el impacto del dolor crónico en las actividades diarias mediante una escala con rango 1-10, mostró una puntuación de 6,3 para los pacientes españoles. En el conjunto de los encuestados, el $50 \%$ se sentía preocupado sobre el efecto que el dolor pudiera tener sobre sus relaciones. Así, el $36 \%$ de los pacientes consideró que su condición tenía un impacto negativo en su familia o amigos, y el $27 \%$ de los encuestados afirmaron sentirse socialmente aislados a causa del dolor (5).

Según datos de la iniciativa Pain Proposal (5) los enfermos de dolor crónico no se sienten comprendidos por la sociedad. Cerca de dos terceras partes de los encuestados (62\%), manifestaron sentir una falta de concienciación y conocimiento de la enfermedad en su entorno, y el $47 \%$ creían que el resto de la gente duda de la existencia real de su dolor.

Todo ello conlleva que el dolor crónico también se asocie con un incremento del riesgo de enfermedades psicológicas como ansiedad o depresión (7), e incluso de suicidio (8). Estudios realizados en España, mostraron que el $47 \%$ de los pacientes con dolor crónico sufren un cuadro depresivo asociado y el $50 \%$ trastornos del sueño $(1,9)$.

\section{DOLOR CRÓNICO EN EL SISTEMA SANITARIO ESPAÑOL}

En España no existe una estrategia nacional del dolor elaborada por el gobierno central. Sólo en el plano de algunas Comunidades Autónomas, como en la Comunidad de Madrid, la Xunta de Galicia, la Generalitat de Catalunya o la Junta de Andalucía, encontramos diversas iniciativas.

La Estrategia en Cuidados Paliativos del Sistema Nacional de Salud, publicada en 2007 por el Ministerio de Sanidad (10), ha conseguido que el tratamiento del dolor crónico de origen oncológico reciba un adecuado apoyo en cuanto a medios e infraestructuras.

En cambio, el tratamiento del dolor crónico no oncológico carece de planes que ofrezcan estrategias de organización y de recursos, así como otros aspectos necesarios que no sean estrictamente farmacológicos (iniciativas de prevención, información, fisioterapias, guías clínicas, etcétera). 


\section{Diagnóstico y manejo del paciente con dolor crónico}

La complejidad para medir el dolor y sus diferentes manifestaciones, dado su carácter subjetivo, pueden dificultar establecer su origen y manejo, por lo que el diagnóstico e identificación de la mejor opción terapéutica puede llevar tiempo $(5,11)$.

Los datos del Pain Proposal (5) reflejaron una situación preocupante. Cerca de la mitad de los entrevistados (49\%) estaban insatisfechos con el tiempo que había pasado hasta haber sido diagnosticados. En nuestro país, más de un tercio de los pacientes (39\%) tardaron más de un año en ser diagnosticados y el $13 \%$ de personas no lograron un diagnóstico. El tiempo medio de demora hasta el diagnóstico en España era, al igual que en Europa, de 2,2 años.

Las consultas por dolor en España son muy comunes, tanto en atención primaria (AP) como en urgencias. La mayoría de ellas (hasta un $80 \%$ ) se resuelven en el ámbito de la atención primaria y el resto se deriva al especialista; si allí no se resuelven entonces pasan a las unidades del tratamiento del dolor (UTD). La escasez de recursos en las UTD, unida a la indefinición en la tipología de pacientes en que está incluida la derivación de las mismas, hace aumentar las listas de espera y alarga el proceso de tratamiento. Ambos factores traen como consecuencia el estancamiento de la enfermedad en los pacientes. Cuando se logra educar a los pacientes de larga duración en el padecimiento y el control del dolor, en lugar de en la desaparición del mismo, estos pasan de nuevo al médico de AP para los controles periódicos. Según la Sociedad Española del Dolor (SED), aproximadamente el $2 \%$ de los pacientes con dolor crónico se encuentran en las UTD, el $83 \%$ en AP y el $15 \%$ en el especialista.

La Clínica para el Dolor de Shouthampton mostró las ventajas del manejo multidisciplinar de los pacientes. Se realizó un radical rediseño del servicio que permitió facilitar los recursos apropiados a los médicos de atención primaria. Así, ellos podían tratar adecuadamente a la mayoría de los pacientes, y derivar sólo unos pocos al especialista, con el que, pieza clave del éxito del servicio, se mantenía una estrecha comunicación. De esta forma, no sólo se logró que el $75 \%$ de los pacientes estuvieran satisfechos sino que supuso también un ahorro en los gastos del $35 \%$ (5).

\section{Tratamiento del dolor crónico}

Es importante que los pacientes reciban el tratamiento más adecuado para su tipo de dolor y circunstancias personales. También es prioritario conseguir que los pacientes reciban el tratamiento adecuado tan pronto como sea posible, ya que resultados de estudios sugieren que los pacientes con una espera de más de 6 meses experimentan deterioro en la calidad de vida, en el bienestar psicológico $\mathrm{y}$ en su estado emocional (12).
Actualmente existen diferentes tratamientos farmacológicos y terapias para el dolor crónico. La escalera analgésica de la Organización Mundial de la Salud (OMS) (13), útil en el dolor crónico de origen nociceptivo, resulta sin embargo de poca utilidad en el tratamiento del dolor de origen neuropático (9), el cual, no responde de forma eficaz a fármacos analgésicos correspondientes al primer escalón (AINE y no-opioides), y requiere el uso de antidepresivos y anticonvulsivantes $(9,14,15)$.

Los resultados del Pain Proposal (5) mostraron que el $38 \%$ del conjunto de europeos, y el $29 \%$ de los españoles consideraban que no recibían el tratamiento adecuado para su dolor. El tiempo medio en conseguir un tratamiento adecuado para el dolor en España era de 1,6 años, ligeramente inferior a la media europea (1,9 años), y cerca de la mitad de los entrevistados (48\%) no estaba conforme con el tiempo necesario para conseguir un tratamiento adecuado (Tabla I).

TABLA I. RESULTADOS DEL PAIN PROPOSAL EN ESPAÑA

Prevalencia del dolor crónico $17 \%$

Porcentaje de pacientes aislados socialmente a causa del dolor*

$27 \%$

Porcentaje de pacientes que tardaron más de un año en ser diagnosticados

Tiempo medio de diagnóstico

2,2 años

Porcentaje de pacientes que consideran recibir un tratamiento no adecuado

$29 \%$

Tiempo medio en recibir un tratamiento adecuado

1,6 años

Porcentaje de pacientes descontentos con el tiempo de espera para recibir un tratamiento adecuado

$48 \%$

Número de consultas médicas realizadas al año a causa del dolor

Media anual de días de baja laboral a causa del dolor*

14 días

Porcentaje de pacientes incapaces de trabajar a causa del dolor*

$21 \%$

Porcentaje de médicos que expresaron su deseo de recibir formación adicional sobre el $\quad 85 \%$ manejo del dolor crónico*

*Datos del conjunto de Europa. 
Por otra parte, los pacientes suelen tener expectativas no realistas respecto a la medicación prescrita (11). Muchos de los tratamientos disponibles sólo logran ligeras mejoras y es raro que se consiga una recuperación total. Los profesionales sanitarios deberían establecer unos objetivos reales en cuanto a las expectativas del tratamiento para conseguir así mejores resultados (16).

La falta de eficacia de los tratamientos puede conllevar otros riesgos. Según la SED, el $29 \%$ de los pacientes suele recurrir a la automedicación para tratar su dolor, con las connotaciones que pueden derivarse en cuanto a mal control, efectos adversos (algunos de ellos importantes al ser los AINE los más utilizados) y los riesgos de interacciones (17).

Junto al abordaje farmacológico en los casos en que este es necesario, está el abordaje con medidas no farmacológicas que pueden tener un impacto positivo en la calidad de vida de estos pacientes.

\section{Acceso a la formación especializada}

El Pain Proposal (18) mostró que una gran mayoría de médicos en Europa cree que una mejora en su formación permitiría un manejo más eficaz del dolor de sus pacientes. El $85 \%$ de los médicos europeos entrevistados expresó su deseo de recibir formación adicional para la identificación, tratamiento y manejo del dolor crónico. Aunque es el médico de AP el que ve al paciente con dolor crónico por primera vez en la mayoría de los casos, sólo el $53 \%$ de los médicos de AP europeos declaró saber manejar este tipo de dolencia (18), menos de la mitad (46 \%) indicó estar seguros de saber qué hacer cuando el tratamiento inicial no es el más adecuado y el dolor persiste (18), mientras el $47 \%$ declaró no saber cuándo cambiar un tratamiento (18).

La falta de guías específicas para el manejo del dolor crónico da lugar a un asesoramiento variable. Un significativo número de médicos indicó no sentir plena confianza en la comprensión y uso de directrices existentes (18).
Las guías a nivel internacional son escasas. La OMS publicó en 2008 unas directrices para el tratamiento del dolor crónico no oncológico (19). A nivel nacional no existen directrices o guías de práctica clínica sobre el tratamiento del dolor crónico. Existen las recomendaciones de la SED para el dolor neuropático y a nivel local, cabe citar la excelente Guía de Actuación Clínica en AP elaborada por el Dr. José de Andrés y Dr. Germán Cerdá desde la UTD de Valencia.

En lo concerniente a la capacitación de los profesionales, hoy en día el abordaje del dolor no se trata como un área independiente. Sólo se estudia en la carrera de Medicina, en la asignatura de Farmacología y en los capítulos reservados al dolor en el estudio de cada enfermedad. Existen también dos postgrados en medicina de AP llamados "Abordaje del dolor oncológico" y "Abordaje del dolor crónico" y cursos de formación continuada sobre cuidados paliativos en pacientes oncológicos. Además, a través de la SED se imparten cursos de especialización sobre el dolor como alternativa privada para suplir el vacío existente en la formación reglada de especialistas en dolor intervencionistas. Aparte de esto, hay iniciativas en formación continuada en las comunidades autónomas, pero no con un enfoque específico al dolor crónico sino al dolor paliativo, o a aspectos farmacológicos del dolor.

\section{CARGA ECONÓMICA DEL DOLOR CRÓNICO}

Aunque el coste del dolor crónico es difícil de calcular, ya que no se dispone de datos globales, se cree que cuesta a Europa más de 300 billones de euros (20) o en torno al 1,5$3 \%$ del PIB $(4,21)$. En España se estima que el coste total (directo e indirecto) que ocasiona el dolor crónico sería de 16.000 millones de euros anuales, lo que supone el 2,5 \% del PIB. Estas cifras deben mirarse, en todo caso, con cautela, ya que en nuestro país tampoco existen estudios globales que lo avalen. En cambio, sí se han valorado en cambio los costes por dolor de diferentes patologías (Tabla II). En España, sólo

TABLA II. CARGA ECONÓMICA DEL DOLOR CRÓNICO EN ESPAÑA

\begin{tabular}{lcccccc}
\hline \multicolumn{1}{c}{ Referencia } & $\begin{array}{c}\text { Año del } \\
\text { coste }\end{array}$ & Patología & $\begin{array}{c}\text { Costes } \\
\text { directos }\end{array}$ & $\begin{array}{c}\text { Costes } \\
\text { indirectos }\end{array}$ & $\begin{array}{c}\text { Costes } \\
\text { totales }\end{array}$ & $\begin{array}{c}\text { Costes } \\
\text { totales }\end{array}$ \\
\hline Badia X, et al. (22) & 2001 & Migraña & 198 & 453 & 651 & \\
Rivera J, et al. (29) & 2006 & Fibromialgia & 3.245 & 6.736 & 9.982 & 301 \\
Sicras-Mainar A, et al. (24) & 2007 & Fibromialgia & 1.677 & 6.977 & 8.654 & 63.526 \\
Loza E, et al. (23) & 2007 & Osteoartritis & 1.297 & 205 & 1.502 & $1.071^{*}$ \\
Rodríguez MJ, et al. (17) & 2004 & Dolor neuropático & 5.064 & & & 504 \\
\hline
\end{tabular}

*> 50 años. Los datos numéricos representan el coste anual por paciente en euros. 
Rev. Soc. Esp. del Dolor, Vol. 21, N. ${ }^{o}$ 1, Enero-Febrero 2014

la migraña supone una carga económica de 1.076 millones de euros anuales, en su mayoría (68\%) debido a costes indirectos (732 millones de $€$ ) (22). Se estima que la osteoartritis de rodilla y cadera supone un coste anual de 4.738 millones de euros (23) (equivalente al 0,5\% del PIB).

Expertos del Pain Proposal consideran que no se están empleando eficazmente los recursos que disponemos para tratar el dolor crónico. Un mejor uso de los mismos permitiría ahorrar dinero al sistema sanitario.

Desde el punto de vista económico, el dolor crónico genera gastos debidos a la atención sanitaria y a pérdidas potenciales de producción. Estas últimas se ven agravadas cuando se demora o no llega a tener lugar una atención adecuada.

La magnitud de los costes indirectos, bien en bajas laborales o en pérdidas de productividad, supera con creces a los costes directos ocasionados por el dolor, lo que sugiere que una mejora de la eficacia de la gestión del dolor podría cosechar grandes recompensas económicas.

La carencia de guías junto con el sistema sanitario fragmentado, que obliga en ocasiones a los pacientes a peregrinar de un especialista a otro durante mucho tiempo en busca de un diagnóstico y tratamiento adecuado, juegan un papel claro en el incremento de los costes que el dolor crónico ocasiona.

Lo que en todo caso nos enseña la experiencia de otros países con datos más acreditados es que el tratamiento de los pacientes con dolor crónico a los primeros síntomas se considera una estrategia rentable, al suponer un ahorro en el gasto presupuestario a medio y largo plazo. En otras palabras, el tratamiento no efectivo a corto plazo de los pacientes con dolor crónico supone un mayor consumo de los recursos a largo plazo.

\section{Costes directos}

Los pacientes con dolor crónico hacen uso del sistema sanitario con más frecuencia que aquellos que no lo padecen, además existe una relación directa entre la gravedad del dolor y el uso de recursos sanitarios $(3,24,25)$. Los datos del National Health and Wellness Survey de España mostraron que los pacientes con dolor crónico severo realizan tres veces más visitas a médicos que aquellos sin dolor (3), casi el doble en el caso de padecer dolor moderado. Similar patrón se observó también en las visitas a urgencias o en las hospitalizaciones (3). Los resultados del Pain Proposal (5) muestran que los europeos de media visitaron 6,8 veces a profesionales sanitarios en el último año, este dato se rebaja a 6,6 veces en el caso de los pacientes españoles.

El manejo inapropiado del paciente y empleo de tratamientos ineficaces pueden originar un aumento de las consultas en AP y especialistas, lo cual, según los expertos del Pain Proposal, conduce a importantes costes sanitarios. Estudios han señalado que son las consultas con profesionales de la salud, particularmente especialistas, las responsables de la mayor parte de los costes directos debidos al dolor crónico (21). En este sentido, varios autores han señalado que la reducción del dolor, mediante el uso de medicamentos y terapias/ dosis adecuadas, se asocia a una reducción de la utilización de recursos sanitarios, en especial de las visitas realizadas $(26-28)$.

\section{Costes indirectos}

Aunque los costes sanitarios directos derivados del manejo del dolor crónico son considerables, esto es sólo una parte del problema cuando se compara con el impacto de los costes indirectos atribuibles al dolor crónico en el conjunto de la economía.

No hay duda que el dolor crónico reduce considerablemente la capacidad laboral. El dolor crónico impone una pesada carga tanto a empleados como a las empresas, ya que la mayoría de los costes indirectos están relacionados con el entorno laboral.

Los europeos con dolor crónico encuestados en el Pain Proposal (5) consideraban que su dolor afectaba de forma negativa a su capacidad para trabajar durante más del $28 \%$ de su jornada laboral. El $21 \%$ manifestaron sentirse incapaces de trabajar, y entre aquellos que sí lo eran, el $61 \%$ declaró que su status laboral se había visto afectado por su enfermedad. Además, el $29 \%$ de los europeos con dolor crónico temen perder su trabajo a causa de su enfermedad. Estudios nacionales mostraron que el $22 \%$ de los pacientes que sufren dolor crónico pierden su empleo, un $8 \%$ sufre cambios de responsabilidad en su puesto de trabajo y un $4 \%$ se ven obligados a cambiar de trabajo (17).

Los gastos que la administración y empresarios soportan son importantes. En España se ha estimado que el $30 \%$ de los pacientes con dolor crónico han estado de baja a lo largo de su vida laboral a consecuencia del dolor (9). Resultados del Pain Proposal (5) mostraron que los europeos con dolor crónico están de baja una media de 14 días al año a causa de su enfermedad. Esta cifra es muy inferior a las mostradas por diversos estudios observacionales en pacientes con dolor neuropático, en especial si se trata de radiculopatías cervicales/lumbares, donde se observaron de media más de 20 días de baja en el periodo de estudio de 12 semanas $(27,28)$.

El Instituto de Estudios Fiscales cifra el impacto del dolor crónico en el mercado laboral en 2.500 millones de euros con 52 millones de jornadas laborales perdidas. 


\section{CAMBIOS NECESARIOS PARA MEJORAR LA GESTIÓN GLOBAL DEL DOLOR CRÓNICO}

-Debemos plantear, sin que esto suponga un incremento del gasto público, la necesidad de crear un plan estratégico nacional sobre el dolor crónico en cuya elaboración colabore la administración, la comunidad científica y las asociaciones de pacientes. Este plan estratégico deberá ahondar en una gestión más eficaz de los recursos, suponer un ahorro a medio y largo plazo y permitir una redistribución más eficiente de estos fondos; además debe contemplar una mayor capacitación y coordinación entre los distintos profesionales médicos para gestionar eficazmente el dolor crónico, y reforzar la educación de los pacientes y la sociedad en general sobre los aspectos más importantes de esta enfermedad de tratamiento tan complejo.

-Es necesario desarrollar un proceso integrado del abordaje del paciente con dolor crónico mediante la implementación de procesos y planes territoriales en los que se establezcan los diferentes niveles de tratamiento (desde la medicina primaria, hasta la clínica del dolor multidisciplinaria), estableciendo el circuito y las estructuras habilitadas para atender al paciente, analizando la situación en cada zona determinada, para establecer recursos adecuados a las necesidades de cada población y caso concreto.

- Incidir en la capacitación de los profesionales, con la puesta en marcha de manera definitiva del área de capacitación específica en dolor y la posibilidad de formarse en una especialidad que cada vez requiere mayores conocimientos y habilidades técnicas.

- Mejorar la coordinación entre la atención primaria y la atención hospitalaria, así como entre los diferentes especialistas de la misma, para gestionar eficazmente el dolor crónico sin tener que llegar a las UTD, donde solo irían los casos que realmente lo necesitaran.

- Reforzar la educación de los pacientes y de la población en general sobre los aspectos más importantes de la enfermedad y hacerles partícipes para fomentar su autonomía.

- Asumir que el tratamiento efectivo y preventivo del dolor crónico es un proceso complejo que incluye aspectos fisiológicos, psicológicos y socioculturales con el fin de incrementar la percepción de control del dolor por parte del paciente y modificar, en ciertos casos, las creencias en torno a su condición y a su enfermedad. Esto podría incidir favorablemente en la calidad de vida del paciente y su familia.

\section{CONCLUSIONES}

Estos datos deberían hacernos reflexionar sobre la necesidad de un plan nacional, una mejora en la formación, divulgación de protocolos, guías específicas y sobre los beneficios que puede traer consigo una mayor coordinación entre la atención primaria y la especializada en el tratamiento del dolor crónico. En las tablas III y IV se resumen las carencias y aquello que funciona en nuestro sistema sanitario.

Un diagnóstico y tratamiento certero y rápido repercutirá en una mejor calidad de vida del paciente y en un ahorro en la carga económica de la enfermedad.

\section{TABLA III. ¿CUÁLES SON LAS CARENCIAS?}

- Falta de un plan estratégico nacional sobre el dolor crónico

- Falta de una visión global e integradora sobre la complejidad a la hora de tratar el dolor crónico

- Carencia de estudios económicos específicos que muestren la carga económica y social que el dolor crónico supone para el sistema sanitario y la sociedad en general

- Carencia de especialización en dolor crónico en la formación reglada de los profesionales de la salud

- Falta de coordinación entre profesionales médicos de la atención primaria y entre los diferentes especialistas de atención especializada, lo que ocasiona a veces un consumo innecesario de recursos sanitarios

- Falta de formación sólida y asociación por parte de los pacientes

- Falta de concienciación social sobre el dolor crónico y la necesidad de su tratamiento específico

TABLA IV. ¿QUÉ FUNCIONA EN NUESTRO SISTEMA SANITARIO?

Desde el punto de vista clínico

- El enfoque que se está dando en los cuidados paliativos después de la publicación de la estrategia, y que debería ampliarse al tratamiento del dolor en general

- La UTD de Valencia con el Dr. José de Andrés, centrada en la AP, pero multidisciplinar (paliativos, formación, guía práctica sobre derivación y fármacos, etc.)

- El programa de atención domiciliaria de la CAM con el que mejoró el conocimiento de la realidad cotidiana de las personas con dolor crónico 
TABLA IV. ¿QUÉ FUNCIONA EN NUESTRO SISTEMA SANITARIO?

\section{En el plano de la administración}

- Gratuidad y universalidad del sistema sanitario español.

- Planes de cuidados paliativos de las distintas comunidades autónomas

- Protocolos de la Xunta (tendente a mejorar la comunicación entre atención primaria y especialistas, y la derivación de los pacientes con dolor crónico)

- Plan Andaluz de Atención a las Personas con Dolor, en el que se resumen los estudios elaborados en España

- Proceso asistencial de abordaje del dolor crónico no oncológico también en Andalucía

\section{CORRESPONDENCIA:}

Antonio Torralba

Coordinadora Nacional de Artritis

C/ Cea Bermúdez, 14 B, 2. ${ }^{\circ} \mathrm{A}$

28003 Madrid

e-mail: presidencia@conartritis.org

\section{BIBLIOGRAFÍA}

1. Breivik H, Collett B, Ventafridda V, et al. Survey of chronic pain in Europe: Prevalence, impact on daily life, and treatment. Eur J Pain 2006;10:287-333.

2. Catala E, Reig E, Artes M, et al. Prevalence of pain in the Spanish population: Telephone survey in 5000 homes. Eur J Pain 2002;6:133-40.

3. Langley PC, Ruiz-Iban MA, Molina JT, et al. The prevalence, correlates and treatment of pain in Spain. J Med Econ 2011;14:367-80.

4. Phillips CJ. Economic burden of chronic pain. Expert Rev Pharmacoecon Outcomes Res 2006;6:591-601.

5. InSites Consulting. Pain Proposal Patient Survey. July-September 2010.

6. Cano A, Miller LR, Loree A. Spouse beliefs about partner chronic pain. J Pain 2009;10:486-92.

7. Poole H, White S, Blake C, et al. Depression in chronic pain patients: Prevalence and measurement. Pain Pract 2009;9:173-80.

8. Tang NK, Crane C. Suicidality in chronic pain: A review of the prevalence, risk factors and psychological links. Psychol Med 2006;36:575-86.

9. Rodríguez MJ. Valoracion de la actitud terapeútica ante el paciente con dolor en las Unidades del Dolor en Espa- ña. Estudio STEP. Revista Sociedad Española del Dolor 2006;8:525-32.

10. www.msps.es/. 2011.

11. Dewar AL, Gregg K, White MI, et al. Navigating the health care system: Perceptions of patients with chronic pain. Chronic Dis Can 2009;29:162-8.

12. Lynch ME, Campbell F, Clark AJ, et al. A systematic review of the effect of waiting for treatment for chronic pain. Pain 2008;136:97-116.

13. World Health Organization. Ed. Geneva: World Hearth Organization 1. Cancer Pain Relief. 2nd ed. 1996.

14. Backonja MM. Defining neuropathic pain. Anesth Analg 2003;97:785-90.

15. National Institute for Health and Clinical Excellence. Neuropathic pain: The pharmacological management of neuropathic pain in adults in non-specialist settings. Clinical Guildeline 96. London: NICE, 2010.

16. Woolf A, Carr A, Frolich J, et al. Investigating the barriers to effective management of musculoskeletal pain: An international survey. Clin Rheumatol 2008;27:1535-42.

17. Rodriguez MJ, García AJ. Costes del dolor neuropático según etiología en las Unidades del Dolor en España. Revista Sociedad Española del Dolor 2007;6:404-15.

18. InSites Consulting. Pain Proposal PCP Survey. July-September 2010.

19. World Health Organisation Steering Group on Pain Guidelines. Scoping Document for WHO Treatmnet Guidelines on Chronic Non-Malignant Pain in Adults 2008. Available at: http://www.who.int/medicines/areas/quality_safety/ Scoping WHOGuide non-malignant pain adults.pdf Last accessed 13.09.10. $20 \overline{11}$.

20. Wenig CM, Schmidt CO, Kohlmann T, et al. Costs of back pain in Germany. Eur J Pain 2009;13:280-6.

21. Mantyselka PT, Kumpusalo EA, Ahonen RS, et al. Direct and indirect costs of managing patients with musculoskeletal pain-challenge for health care. Eur J Pain 2002;6:141-8.

22. Badia X, Magaz S, Gutierrez L, et al. The burden of migraine in Spain: Beyond direct costs. Pharmacoeconomics 2004;22:591-603.

23. Loza E, Lopez-Gomez JM, Abasolo L, et al. Economic burden of knee and hip osteoarthritis in Spain. Arthritis Rheum 2009;61:158-65.

24. Sicras-Mainar A, Rejas J, Navarro R, et al. Treating patients with fibromyalgia in primary care settings under routine medical practice: A claim database cost and burden of illness study. Arthritis Res Ther 2009;11:R54.

25. Blyth FM, March LM, Brnabic AJ, et al. Chronic pain and frequent use of health care. Pain 2004;111:51-8.

26. Perez C, Saldana MT, Navarro A, et al. Trigeminal neuralgia treated with pregabalin in family medicine settings: Its effect on pain alleviation and cost reduction. J Clin Pharmacol 2009;49:582-90.

27. Navarro A, Saldana MT, Perez C, et al. A cost-consequences analysis of the effect of pregabalin in the treatment of peripheral neuropathic pain in routine medical practice in primary care settings. BMC Neurol 2011;11:7.

28. Saldana MT, Navarro A, Perez C, et al. A cost-consequences analysis of the effect of pregabalin in the treatment of painful radiculopathy under medical practice conditions in primary care settings. Pain Pract 2010;10:31-41.

29. Rivera J, Rejas J, Esteve-Vives J, et al. Resource utilisation and health care costs in patients diagnosed with fibromyalgia in Spain. Clin Exp Rheumatol 2009;27:S39-S45. 